\title{
Growth in CHARGE association
}

\author{
K Blake, J M W Kirk, E Ur
}

\begin{abstract}
Growth data is shown from 44 children (aged 0.5-18 years; 27 boys, 17 girls) with CHARGE association. Children had a normal birth weight and length, but in infancy there was a fall in centiles. Children remained small and there was pubertal delay in boys. Close monitoring of growth with early detection and intervention is recommended.
\end{abstract}

(Arch Dis Child 1993; 68: 508-509)

CHARGE association is a non-random constellation of congenital anomalies consisting of Colobomata of the eye, Heart defect, choanal Atresia, Retardation of growth and development, Genital hypoplasia, and Ear abnormalities. Since the early description by Hall $e t a l$ in $1979^{\prime}$ it is apparent that facial palsy, renal anomalies, orofacial clefts, and tracheooesophageal atresia/fistula frequently accompany the main features. ${ }^{2}$ Many clinical manifestations have been described in detail, although no large studies concerning growth in this condition have previously been published. As the number of children attending a tertiary referral centre in London far exceeds any other published series we felt it appropriate to describe and tabulate growth patterns among this group.

\section{Patients and methods}

Auxological data was available on 44 children (aged 0.5-18 years; 27 boys, 17 girls) with CHARGE association attending the Hospital for Sick Children at Great Ormond Street, London. The diagnosis of CHARGE association was made on the presence of at least four of the six features prefixed by the letters of the acronym, and was also confirmed in most cases by a consultant geneticist. Growth data was obtained from hospital inpatient and outpatient medical records.

Mean (SD) birth weight and length were documented for both boys and girls, as were subsequent weight and height data for the first two years measured at intervals of three months. Data were analysed at $3,5,7,10$ years $( \pm 6$ months) for both sexes, and in addition in the boys at 12, 14, and 16 years. These were plotted on standard Tanner and Whitehouse growth curves. $^{3}$

Royal Free Hospital, London K Blake

St Bartholomew's Hospital, London $\mathrm{J} M$ W Kirk

EUr

Correspondence to: Dr J M W Kirk, King's College Hospital, Denmark Hill, London SE5 9RS

Accepted 24 December 1992

Results (see figs 1 and 2)

Mean birth weights and lengths for all patients were on or above the 10th centile, but if birth weights of the infants born prematurely $(<37$ weeks' gestation, $n=3$ ) were excluded then the means were on or above the 50 th centile. During the first 12 months of life the mean weights and lengths for both sexes fell below the third centile.
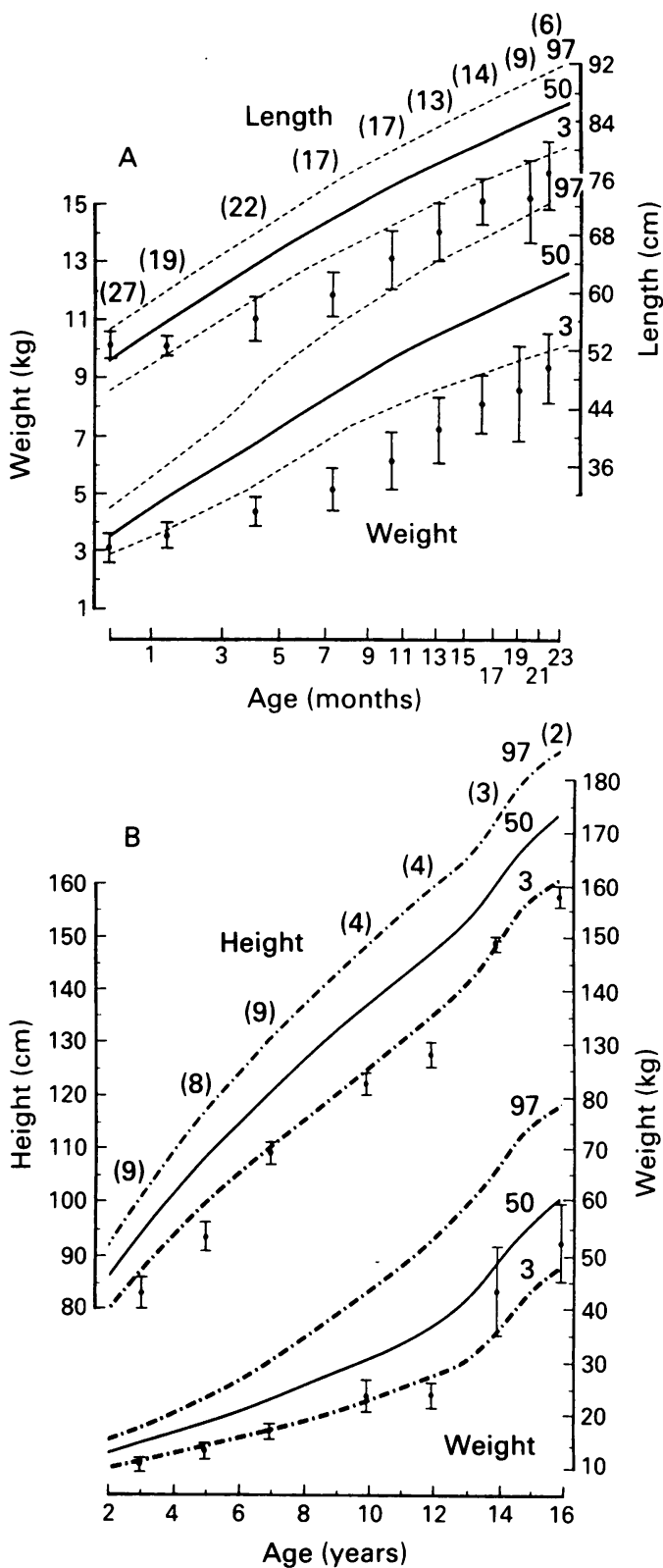

Figure 1 Mean $(S D)$ length/height and weight for boys with $C H A R G E$ association compared with normal boys $(A)$ in the first two years of life and $(B)$ aged 2-16 years. Number of children shown in parentheses.

There was catch-up growth in the preschool years (more so in the girls than in the boys). One child had documented growth hormone insufficiency and his growth data has been omitted.

All girls are prepubertal, and of the five boys spontaneous puberty.

Gastro-oesophageal reflux diagnosed by barium swallow or $\mathrm{pH}$ studies was present in 15 of the 26 children over 1 year of age who were failing to thrive (height $<3$ rd centile), but in only one child with normal growth. Facial palsy was 

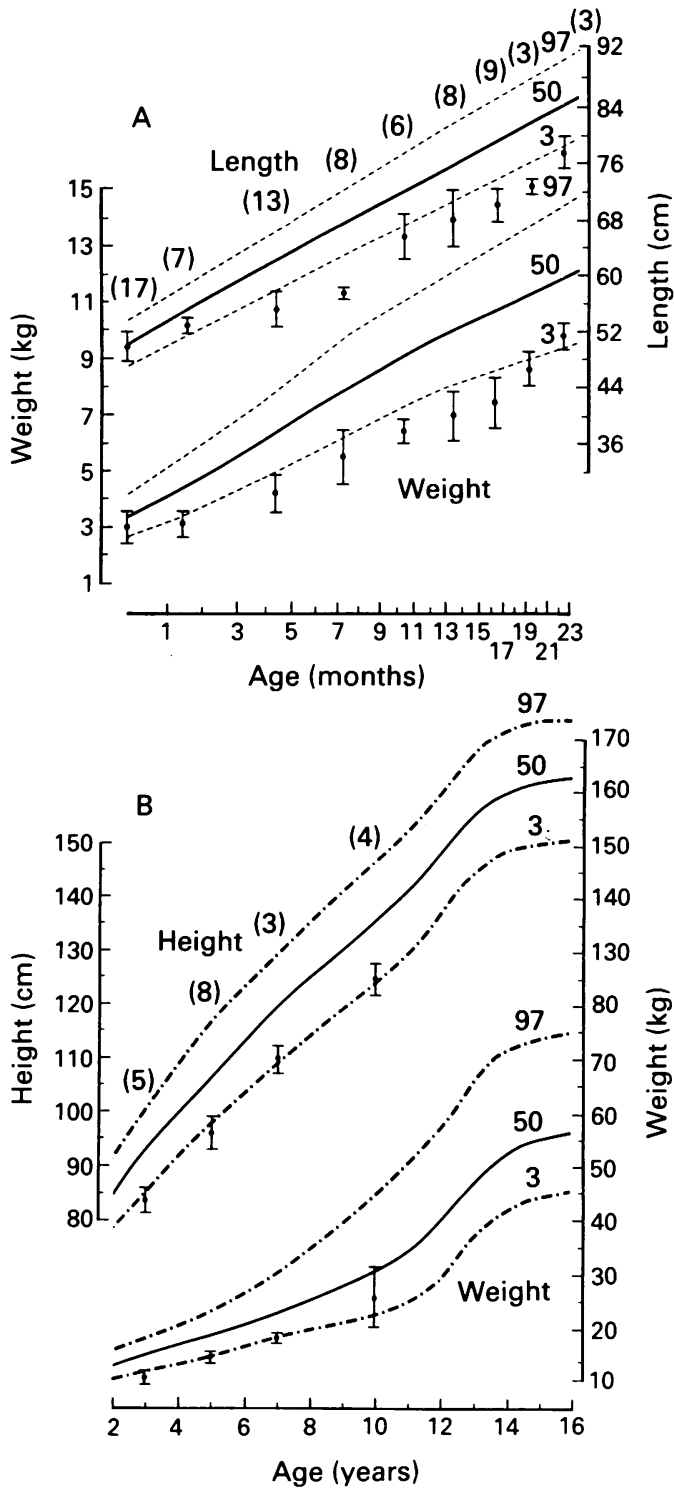

Figure 2 Mean $(S D)$ length/height and weight for girls with $C H A R G E$ association compared with normal girls $(A)$ in the first two years of life and $(B)$ aged 2-16 years. Number of children shown in parentheses.

also significantly associated with failure to thrive, with $60 \%$ of patients affected.

\section{Discussion}

Children with CHARGE association have a normal birth weight and length and it is during infancy that there is the most marked decline away from the centiles. Previous work with microlaryngobronchoscopy techniques has shown that structural anomalies of the larynx and pharynx as well as pharyngeal incoordination is a common finding in many of the children with CHARGE. ${ }^{4}$ This, in addition to the high occurrence of gastro-oesophageal reflux, may account for the difficulties the children have initially with feeding, and in introduction of solid food to their diet. Those children who maintained their weight in the early months were ones who had nutritional intervention (usually in the form of gastrostomy tube feeding). Growth hormone insufficiency was uncommon. There is, however, evidence to suggest that the pubertal delay in boys is secondary to hypogonadotrophic hypogonadism due to gonadotrophin releasing hormone deficiency, ${ }^{5}$ occasionally in association with anosmia (Kallmann's syndrome).

Although the numbers of children in the study are insufficient to extrapolate into growth curves, the purpose of our work is to try and identify those children who have a problem with their growth so that it may be possible to institute investigation and treatment at an early stage. Many of these children are now surviving and it is important to address long term problems from an early stage in management, using a multidisciplinary approach. The early pattern of growth may be related to a combination of poor nutrition and extensive hospitalisation for major surgery. However, the limited catch-up growth in stature is significant and we feel this phenomenon warrants further investigation. It was surprising how few of the children in our series were attending a growth or endocrine clinic.

The study was conducted at the Hospital for Sick Children, Great Ormond Street, London.

1 Hall BD. Choanal atresia and associated multiple anomalies. F Pediatr 1981; 95: 395-8.

Oley CA, Baraitser M, Grant DB. A reappraisal of CHARGE association. 7 Med Genet 1988; 25: 147-56.

3 Tanner JM, Whitehouse RH, Takaishi M. Standards from birth to maturity for height, weight, height velocity, and birth to maturity for height, weight, height velocity, and weight velocity: British

4 Blake KD, Russell-Eggitt IM, Morgan DW, Ratcliffe JM Wyse RKH. Who's in CHARGE? Multidisciplinary management of patients with CHARGE association. Arch Dis Child 1990; 65: 217-23.

5 August PA, Rosenbaum KN, Friendly D. Hypopituitarism and the CHARGE association. F Pediatr 1983; 103: 424-5. 